\title{
Distinctive Mediastinal Appearance in Chest Radiograph of a Patient with Total Anomalous Pulmonary Venous Connection
}

Firdouse $\mathrm{M}^{1}$, Agarwal $\mathrm{A}^{1}$, Predescu $\mathrm{D}^{2}$, Gilleland $\mathrm{J}^{2}$ and Mondal $\mathrm{T}^{2 *}$

${ }^{1}$ Faculty of Health science, McMaster University, Hamilton, ON, Canada

${ }^{2}$ Department of Pediatrics, McMaster Children's Hospital, McMaster University, Hamilton, ON, Canada

\begin{abstract}
Despite technological advancements, diagnosis of total anomalous pulmonary venous connection (TAPVC) can be challenging in neonates and infants, particularly in asymptomatic cases. We report a case of a 22-month-old boy who presented to the Emergency department with a history of intermittent fever and cough, initially diagnosed as pneumonia. The presence of an enlarged mediastinal mass was noted in a chest radiograph and was interpreted as a potential malignancy. However, the presence of a supracardiac type of TAPVC with significant right-sided cardiac dilation and a large unobstructed ascending vertical vein was confirmed by echocardiogram. Elective and uneventful surgical correction was performed with subsequent follow-up to confirm cardiac stability and normalization. This case presents the "snowman" or "figure-of-eight" appearance characteristic of TAPVC. Moreover, our patient's chest radiograph is suggestive of a well-defined, smooth and linear vascular shadow with the presence of a normal lung parenchyma along with increased pulmonary vascularity indicating a left-sided vertical vein in keeping with the supracardiac type of TAPVC. Not commonly reported in previous literature, these unique radiographic findings may hold significant value for pediatricians and imaging specialists as a diagnostic tool for the supracardiac variant of this congenital pathology.
\end{abstract}

Keywords: Total anomalous pulmonary venous connection; Supracardiac; Diagnosis; Mediastinal mass; Radiographic findings; X-ray

\section{Introduction}

Identification of a superior mediastinal shadow in a child admitted in the intensive care unit is always of grave concern and warrants urgent attention. There are many differential diagnoses in such a case, some of which are life threatening and others, which are less critical. Total anomalous pulmonary venous connection (TAPVC) occurs in approximately $2 \%$ of all children born with congenital heart defects [1]. It is a rare malformation of the heart in which the pulmonary veins (PV) carrying oxygenated blood drain directly into the deoxygenated systemic venous circulation rather than the left atrium (LA) [2]. Consequently, the presence of an obligatory atrial septal defect (ASD) allows the right-to-left shunting of mixed blood [3].

Craig et al. [4] was the first to classify TAPVC into four groups based on the site of drainage of the PV: (i) Supracardiac: All the PV meet at the confluence behind the LA and drain via an ascending vertical vein into the innominate vein, superior vena cava (SVC) or the azygous vein. This is the most common form and occurs in about $50 \%$ of all TAPVC patients; (ii) Cardiac: All the PVs drain directly into the coronary sinus or right atrium (RA). This occurs in about $30 \%$ of all TAPVC patients; (iii) Infracardiac: All the PV meet at the confluence and drain via a descending vertical vein into the hepatic or portal vein below the diaphragm. This occurs in about $15 \%$ of all TAPVC patients; (iv) Mixed: Involves a combination of the aforementioned variants. This occurs in about $5 \%$ of all TAPVC patients [1]. TAPVC can also be classified by the presence or absence of an obstruction in the pulmonary circuit [3]. For instance, the ascending vertical vein in a supracardiac TAPVC could be trapped and compressed in a vise-like manner between the left main bronchus and the left pulmonary artery. The lumen of the descending vertical vein in an infracardiac TAPVC also narrows significantly as it passes through the diaphragm to drain into the hepatic or portal vein. Moreover, the hepatic parenchyma itself poses a high resistance to pulmonary venous flow. Obstruction can also be caused by the presence of a restrictive ASD, which hinders the vital right-to-left shunting [5].
Although children with obstructive TAPVC always present with pulmonary edema, cyanosis, dyspnea and a lack of murmur $[6,7]$, unobstructive TAPVC may or may not manifest itself early in the neonatal course. Cases are frequently misdiagnosed as having respiratory tract infection or pulmonary disease [1]. Chest radiography (CXR), electrocardiography and transthoracic echocardiography are non-invasive methods typically used to screen children with a suspected TAPVC. More recently, computer tomography (CT)assisted angiography has also become a reliable tool in the preoperative evaluation of cardiac anatomy in TAPVC patients [8]. Despite technological advancements, diagnosis of TAPVC is especially challenging in neonates and infants with developing mediastinal structures, as it is highly dependent on the clarity and resolution of the images obtained. Although the aforementioned symptoms are commonly identified in majority of the TAPVC cases, there are also asymptomatic or clinically undiagnosed presentations. The infrequency of these cases and difficulties in making clinical findings associated with their diagnosis makes our case of special interest to pediatricians and imaging specialists. While this condition has been documented to present as a "snowman" or "figure-of-eight" appearance in CXRs, in this case report, we present a novel mug-shaped appearance as a new diagnostic radiographic feature for diagnosing supracardiac TAPVC.

\section{Case Report}

We present a 22-month-old boy born at term with a birth weight

*Corresponding author: Dr. Tapas Mondal, Department of Pediatrics, McMaster University, 1280 Main Street West, Hamilton, ON, L8S 4K1, Canada, Tel: (905) 521-2100 x75259; E-mail: mondalt@mcmaster.ca

Received August 08, 2013; Accepted September 10, 2013; Published Septembe 12, 2013

Citation: Firdouse M, Agarwal A, Dragos Predescu, Gilleland J, Mondal T (2013) Distinctive Mediastinal Appearance in Chest Radiograph of a Patient with Total Anomalous Pulmonary Venous Connection. Health Care Current Reviews 1: 102. doi: 10.4172/2375-4273.1000102

Copyright: (c) 2013 Firdouse M, et al. This is an open-access article distributed under the terms of the Creative Commons Attribution License, which permits unrestricted use, distribution, and reproduction in any medium, provided the original author and source are credited. 
Citation: Firdouse M, Agarwal A, Dragos Predescu, Gilleland J, Mondal T (2013) Distinctive Mediastinal Appearance in Chest Radiograph of a Patient with Total Anomalous Pulmonary Venous Connection. Health Care Current Reviews 1: 102. doi: 10.4172/2375-4273.1000102

of $3082 \mathrm{~g}$. The patient presented with a history of intermittent fever and cough at the Emergency Room at McMaster Children's Hospital. Cardiac auscultation was normal. The patient was clinically diagnosed with pneumonia and treated with antibiotic therapy without improvement. The patient had an oxygen saturation of $85 \%$ to $87 \%$ on room air. A subsequent CXR identified the presence of an enlarged mediastinal mass (Figure 1a). The mass was further investigated by a chest CT without contrast revealed an abnormal mediastinal vasculature shadow suggesting a normal variation of leftsided SVC and coronary sinus. A non-sedatedechocardiogram was then performed to confirm the abnormal vasculature, and revealed the presence of an unobstructed supracardiac type of TAPVC with all PV draining into a confluence behind the LA, and an ascending vertical vein joining the brachiocephalic vein to drain into the RA through the SVC. A large ASD was also present with RA and right ventricle (RV) dilation with good biventricular function. The patient had an elective and uneventful repair in 2 weeks. Postoperative echocardiograms revealed good biventricular systolic function, adequate LV filling, no residual ASD, and an unobstructed pulmonary venous return to the LA, although mild dilation and minor hypertrophy of the RV was noted. The patient had a normal oxygen saturation of $97 \%$ on room air. The postoperative CXR no longer had the snowman appearance. The patient was reported to be clinically stable from a cardiac perspective. A follow up echocardiogram at 6 months confirmed excellent surgical correction with normal flow pattern across the PV and normalization of the right-sided chambers. The child is now 5 years old and remains in sinus rhythm and good cardiac status.

\section{Discussion}

Interestingly, the largely acute onset of the aforementioned symptoms in our case led to the unexpected diagnosis of a supracardiac type TAPVC, which is known to present with chronic symptoms. Initial inspection of the preoperative CXR showed a questionable mediastinal widening whose differential diagnosis often includes lymphomas, neurogenic, bronchiogenic or mesenchymal tumors, teratomas and thymic lesions [9]. Due to this acute presentation at this age, congenital heart abnormalities such as pulmonary atresia with ventricular septal defect and major aortopulmonary collateral arteries were overlooked. A non-sedated CT without contrast was performed to investigate the possibility of a malignancy, considering the patient's history suggestive of acute respiratory infection. While the results indicated an enlargement of the SVC, brachiocephalic and coronary sinus, no mediastinal mass was observed. Although the CXR had a crude snowman or figure-of-

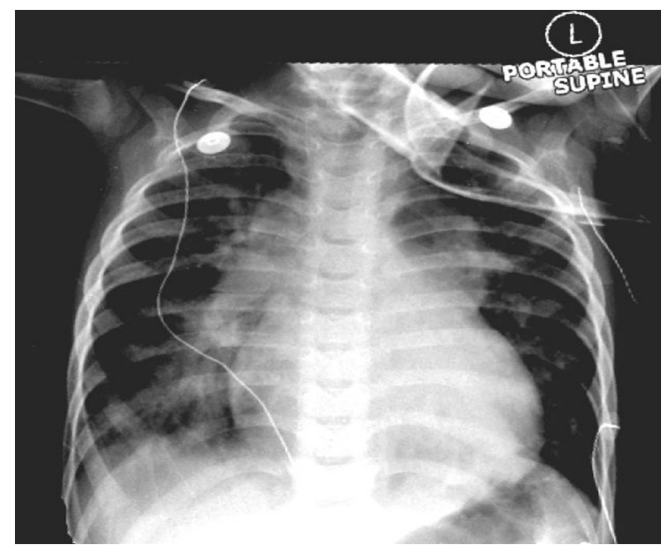

Figure 1a: Preoperative chest radiograph. eight appearance [10], which is often associated with supracardiac TAPVC, more intricate details were identified upon closer investigation. The presence of a well-defined paravertebral vascular shadow implies the presence of an ascending vertical vein to the brachiocephalic vein. While this vascular shadow might be interpreted as a left-sided superior vena cava on a CXR or axial CT (Figure 1b); it does not have the same position as a vertical vein of a supracardiac type TAPVC. Moreover, CXRs of mediastinal masses usually manifest themselves as opacities with asymmetrical and lateral invasion into a hemithorax, which are most dense centrally with irregular outer margins, not conforming to any linear vascular structure. There could be calcification and coarseheterogeneity, which was absent in our case [9]. The presence of normal lung parenchyma with a lateral margin formed by a vascular shadow is suggestive of an ascending vertical vein. Furthermore, a smooth (with no corrugated appearance) bilateral widening of the upper cardiac silhouette, along with reduced oxygen saturation and the presence of increased pulmonary vascularity, almost confirms a case of TAPVC. These features resemble a mug shape in a CXR (Figure 2), where the handle represents the ascending vertical vein. Based on our knowledge, this radiographic feature has not been previously described.

\section{Conclusion}

The novelty of this case lies in the presentation of a new CXR finding which is strongly predictive of a supracardiac TAPVC as a primary differential diagnosis. One of the most common causes of upper mediastinal widening in a CXR is mediastinal malignancy. However, the presence of normal lung parenchyma between the cardiac silhouette and a smooth vertical vascular shadow along with increased pulmonary vascularity should raise the suspicion of the presence of a vertical vein as in supracardiac TAPVC as a differential diagnosis. While the snowman or figure-of-eight presentation in CXRs is classic in TAPVC, a mug-shaped appearance with intervening normal lung parenchyma and reduced oxygen saturation may be considered more indicative of a left-sided supracardiac TAPVC, as evident in our case. Although patients vary in presentation and might not always present with symptoms such as shortness of breath and fatigue, the presence of such radiographic findings is almost confirmatory of supracardiac TAPVC. Thus, immediate identification of such radiographic features can improve the timely diagnosis, prognosis and outcome of patients with TAPVC.

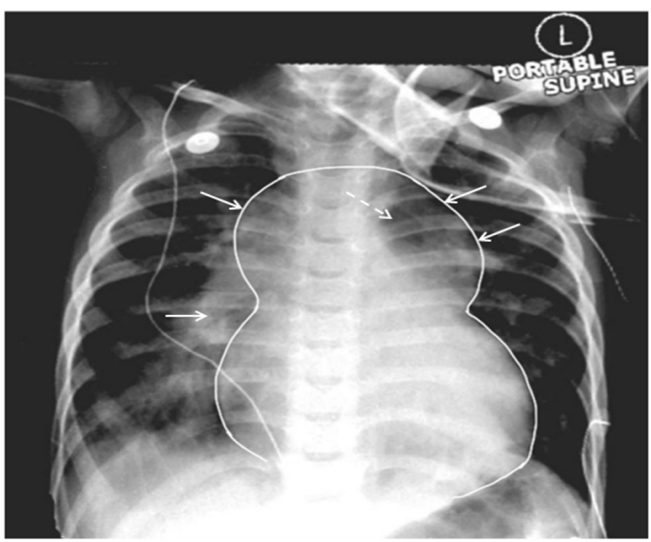

Figure 1b: The solid arrows represent the smooth border of the right mediastinum (right upper arrow) and increased pulmonary vascularity (righ lower arrow). The solid arrows on the left demarcate the smooth and vertical vascular shadow surrounded by normal lung parenchyma (dashed arrow) in the snowman 
Citation: Firdouse M, Agarwal A, Dragos Predescu, Gilleland J, Mondal T (2013) Distinctive Mediastinal Appearance in Chest Radiograph of a Patient with Total Anomalous Pulmonary Venous Connection. Health Care Current Reviews 1: 102. doi: 10.4172/2375-4273.1000102

Page 3 of 3

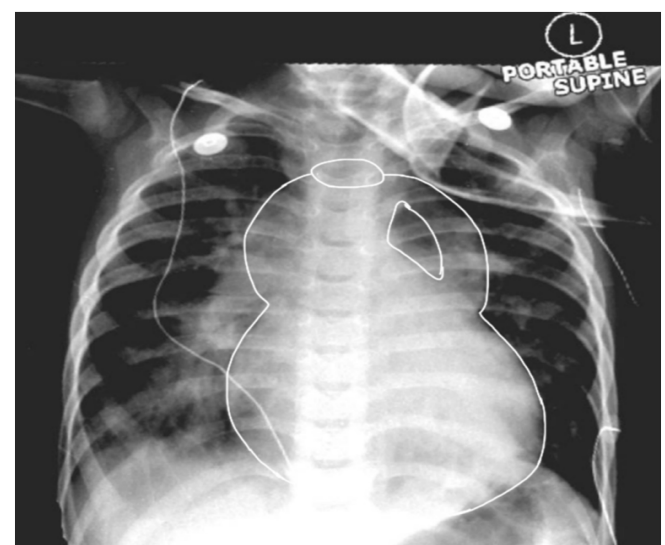

Figure 2: Distinctive jug shape appearance with a handle (the vertical vein) in the chest radiograph

\section{References}

1. Bharati S, Lev M (1973) Congenital anomalies of the pulmonary veins. Cardiovasc Clin 5: 23-41.

2. Delisle G, Ando M, Calder AL, Zuberbuhler JR, Rochenmacher S, et al. (1976)
Total anomalous pulmonary venous connection: Report of 93 autopsied cases with emphasis on diagnostic and surgical considerations. Am Heart J 91: 99122.

3. Stein $P$ (2007) Total anomalous pulmonary venous connection. AORN J 85 : 509-520.

4. Craig JM, Darling RC, Rothney WB (1957) Total pulmonary venous drainage into the right side of the heart; report of 17 autopsied cases not associated with other major cardiovascular anomalies. Lab Invest 6: 44-64.

5. Amplatz K, Moller J (1993) Radiology of Congenital Heart Disease. MosbyYearbook, St. Louis.

6. Emmel M, Sreeram N (2004) Total Anomalous Pulmonary Vein Connection: Diagnosis, Management, and Outcome. Curr Treat Options Cardiovasc Med 6: 423-429.

7. James CL, Keeling JW, Smith NM, Byard RW (1994) Total anomalous pulmonary venous drainage associated with fatal outcome in infancy and early childhood: an autopsy study of 52 cases. Pediatr Pathol 14: 665-678.

8. Shen Q, Pa M, Hu X, Wang J (2013) Role of plain radiography and CT angiography in the evaluation of obstructed total anomalous pulmonary venous connection. Pediatr Radiol 43: 827-835.

9. Kaiser GL (2012) Symptoms and Signs in Pediatric Surgery. Springer, Heidelberg New York Dordrecht, London.

10. Snellen HA, Albers FH (1952) The clinical diagnosis of anomalous pulmonary venous drainage. Circulation 6: 801-816. 